\title{
Relating Graph Thickness to Planar Layers and Bend Complexity*
}

\author{
Stephane Durocher ${ }^{\dagger 1}$ and Debajyoti Mondal ${ }^{2}$ \\ 1 Department of Computer Science, University of Manitoba, Winnipeg, Canada \\ durocher@cs.umanitoba.ca \\ 2 Department of Computer Science, University of Manitoba, Winnipeg, Canada \\ jyoti@cs.umanitoba.ca
}

\begin{abstract}
The thickness of a graph $G=(V, E)$ with $n$ vertices is the minimum number of planar subgraphs of $G$ whose union is $G$. A polyline drawing of $G$ in $\mathbb{R}^{2}$ is a drawing $\Gamma$ of $G$, where each vertex is mapped to a point and each edge is mapped to a polygonal chain. Bend and layer complexities are two important aesthetics of such a drawing. The bend complexity of $\Gamma$ is the maximum number of bends per edge in $\Gamma$, and the layer complexity of $\Gamma$ is the minimum integer $r$ such that the set of polygonal chains in $\Gamma$ can be partitioned into $r$ disjoint sets, where each set corresponds to a planar polyline drawing. Let $G$ be a graph of thickness $t$. By Fáry's theorem, if $t=1$, then $G$ can be drawn on a single layer with bend complexity 0 . A few extensions to higher thickness are known, e.g., if $t=2$ (resp., $t>2$ ), then $G$ can be drawn on $t$ layers with bend complexity 2 $($ resp., $3 n+O(1))$.

In this paper we present an elegant extension of Fáry's theorem to draw graphs of thickness $t>2$. We first prove that thickness- $t$ graphs can be drawn on $t$ layers with $2.25 n+O(1)$ bends per edge. We then develop another technique to draw thickness- $t$ graphs on $t$ layers with reduced bend complexity for small values of $t$, e.g., for $t \in\{3,4\}$, the bend complexity decreases to $O(\sqrt{n})$. Previously, the bend complexity was not known to be sublinear for $t>2$. Finally, we show that graphs with linear arboricity $k$ can be drawn on $k$ layers with bend complexity $\frac{3(k-1) n}{(4 k-2)}$.
\end{abstract}

1998 ACM Subject Classification I.3.5 Computational Geometry and Object Modeling, G.2.2 Graph Theory

Keywords and phrases Graph Drawing, Thickness, Geometric Thickness, Layers, Bends

Digital Object Identifier 10.4230/LIPIcs.ICALP.2016.10

\section{Introduction}

A polyline drawing of a graph $G=(V, E)$ in $\mathbb{R}^{2}$ maps each vertex of $G$ to a distinct point, and each edge of $G$ to a polygonal chain. Many problems in VLSI layout and software visualization are tackled using algorithms that produce polyline drawings. For a variety of practical purposes, these algorithms often seek to produce drawings that optimize several drawing aesthetics, e.g., minimizing the number of bends, minimizing the number of crossings, etc. In this paper we examine two such parameters: bend complexity and layer complexity.

The thickness of a graph $G$ is the minimum number $\theta(G)$ such that $G$ can be decomposed into $\theta(G)$ planar subgraphs. Let $\Gamma$ be a polyline drawing of $G$. Then the bend complexity

\footnotetext{
* A full version of the paper is available online at [11].

$\dagger$ Work of the author is supported in part by the Natural Sciences and Engineering Research Council of Canada (NSERC).
} 


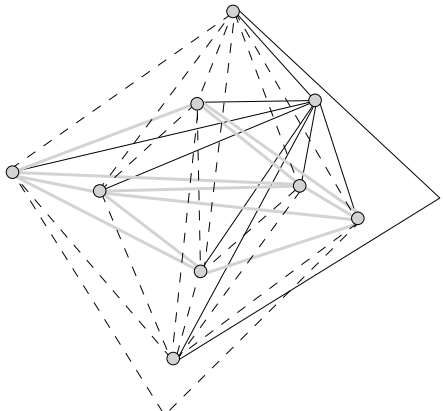

(a)

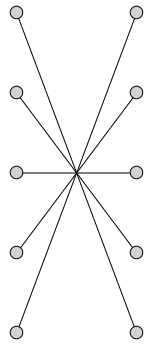

(b)

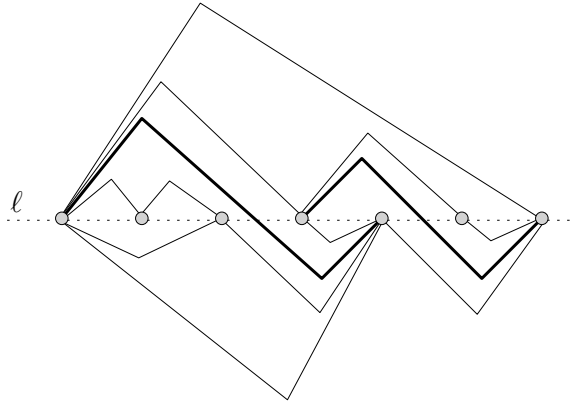

(c)

Figure 1 (a) A polyline drawing of $K_{9}$. (b) A drawing of a matching of size 5. (c) A monotone topological book embedding of some graph. The edges that crosses the spine $\ell$ are shown in bold.

of $\Gamma$ is the minimum integer $b$ such that each edge in $\Gamma$ has at most $b$ bends. A set of edges $E^{\prime} \subseteq E$ is called a crossing-free edge set in $\Gamma$, if the corresponding polygonal chains correspond to a planar polyline drawing, i.e., no two polylines that correspond to a pair of edges in $E^{\prime}$ intersect, except possibly at their common endpoints. The layer complexity of $\Gamma$ is the minimum integer $t$ such that the edges of $\Gamma$ can be partitioned into $t$ crossing-free edge sets. Figure 1(a) illustrates a polyline drawing of $K_{9}$ on 3 layers with bend complexity 1. At first glance the layer complexity of $\Gamma$ may appear to be related to the thickness of $G$. However, the layer complexity is a property of the drawing $\Gamma$, while thickness is a graph property. The layer complexity of $\Gamma$ can be arbitrarily large even when $G$ is planar, e.g., consider the case when $G$ is a matching and $\Gamma$ is a straight-line drawing, where each edge crosses all the other edges; see Figure 1(b).

The layer complexity of a thickness- $t$ graph $G$ is at least $t$, and every $n$-vertex thickness- $t$ graph admits a drawing on $t$ layers with bend complexity $O(n)$ [20]. The problem of drawing thickness- $t$ graphs on $t$ planar layers is closely related to the simultaneous embedding problem, where given a set of planar graphs $G_{1}, \ldots, G_{t}$ on a common set of vertices, the task is to compute their planar drawings $D_{1}, \ldots, D_{t}$ such that each vertex is mapped to the same point in the plane in each of these drawings. Figure 1(a) can be considered to be a simultaneous embedding of three given planar graphs.

\subsection{Related Work}

Graphs with low thickness admit polyline drawings on few layers with low bend complexity. If $\theta(G)=1$, then by Fáry's theorem [16], $G$ admits a drawing on a single layer with bend complexity 0. Every pair of planar graphs can be simultaneously embedded using two bends per edge $[15,17]$. Therefore, if $\theta(G)=2$, then $G$ admits a drawing on two layers with bend complexity 2. The best known lower bound on the bend complexity of such drawings is one [10]. Duncan et al. [9] showed that graphs with maximum degree four can be drawn on two layers with bend complexity 0 . Wood [21] showed how to construct drawings on $O(\sqrt{m})$ layers with bend complexity 1 , where $m$ is the number of edges in $G$.

Given an $n$-vertex planar graph $G$ and a point location for each vertex in $\mathbb{R}^{2}$, Pach and Wenger [20] showed that $G$ admits a planar polyline drawing with the given vertex locations, where each edge has at most $120 n$ bends. They also showed that $\Omega(n)$ bends are sometimes necessary. Badent et al. [1] and Gordon [18] independently improved the bend complexity to $3 n+O(1)$. Consequently, for $\theta(G) \geq 3$, these constructions can be used to draw $G$ on $\theta(G)$ layers with at most $3 n+O(1)$ bends per edge. 
A rich body of literature $[3,4,12,13]$ examines geometric thickness, i.e., the maximum number of planar layers necessary to achieve 0 bend complexity. Dujmović and Wood [7] proved that $\lceil k / 2\rceil$ layers suffice for graphs of treewidth $k$. Duncan [8] proved that $O(\log n)$ layers suffice for graphs with arboricity two or outerthickness two, and $O(\sqrt{n})$ layers suffice for thickness-2 graphs. Dillencourt et al. [6] proved that complete graphs with $n$ vertices require at least $\lceil(n / 5.646)+0.342\rceil$ and at most $\lceil n / 4\rceil$ layers.

\subsection{Our Results}

The goal of this paper is to extend our understanding of the interplay between the layer complexity and bend complexity in polyline drawings.

We first show that every $n$-vertex thickness- $t$ graph admits a polyline drawing on $t$ layers with bend complexity $2.25 n+O(1)$, improving the $3 n+O(1)$ upper bound derived from $[1,18]$. We then give another drawing algorithm to draw thickness- $t$ graphs on $t$ layers, which improves the bend complexity for smaller values of $t$, e.g., for graphs with $t \in\{3,4\}$, it reduces the bend complexity to $O(\sqrt{n})$. No such sublinear upper bound on the bend complexity was previously known for $t>2$. Finally, we show that every $n$-vertex graph with linear arboricity $k \geq 2$ admits a polyline drawing on $k$ layers with bend complexity $\frac{3(k-1) n}{(4 k-2)}$, where the linear arboricity of a graph $G$ is the minimum number of linear forests (i.e., each connected component is a path) whose union is $G$.

The rest of the paper is organized as follows. We start with some preliminary definitions and results (Section 2). In the subsequent section (Section 3) we present two constructions to draw thickness- $t$ graphs on $t$ layers. Section 4 presents the results on drawing graphs of bounded arboricity. Finally, Section 5 concludes the paper pointing out the limitations of our results and suggesting directions for future research.

\section{Technical Details}

In this section we describe some preliminary definitions, and review some known results.

Let $G=(V, E)$ be a planar graph. A monotone topological book embedding of $G$ is a planar drawing $\Gamma$ of $G$ that satisfies the following properties.

$\mathbf{P}_{1}$ : The vertices of $G$ lie along a horizontal line $\ell$ in $\Gamma$. We refer to $\ell$ as the spine of $\Gamma$.

$\mathbf{P}_{2}$ : Each edge $(u, v) \in E$ is an $x$-monotone polyline in $\Gamma$, where $(u, v)$ either lies on one side of $\ell$, or crosses $\ell$ at most once.

$\mathbf{P}_{3}$ : Let $(u, v)$ be an edge that crosses $\ell$ at point $d$, where $u$ appears before $v$ on $\ell$. Let $u, \ldots, d, \ldots, v$ be the corresponding polyline. Then the polyline $u, \ldots, d$ lies above $\ell$, and the polyline $d, \ldots, v$ lies below $\ell$.

Figure 1(c) illustrates a monotone topological book embedding of a planar graph. Let $G_{1}=\left(V, E_{1}\right)$ and $G_{2}=\left(V, E_{2}\right)$ be two graphs on a common set of vertices. A simultaneous embedding $\Gamma$ of $G_{1}$ and $G_{2}$ consists of their planar drawings $D_{1}$ and $D_{2}$, where each vertex is mapped to the same point in the plane in both $D_{1}$ and $D_{2}$. Erten and Kobourov [15] showed that every pair of planar graphs admit a simultaneous embedding with at most three bends per edge. Giacomo and Liotta [17] observed that by using monotone topological book embeddings Erten and Kobourov's [15] construction can achieve a drawing with two bends per edge. Here we briefly recall this drawing algorithm. Without loss of generality assume that both $G_{1}$ and $G_{2}$ are triangulations. Let $\pi_{i}$, where $1 \leq i \leq 2$, be a vertex ordering that corresponds to a monotone topological book embedding of $G_{i}$. Let $P_{i}$ be the corresponding spinal path, i.e., a path that corresponds to $\pi_{i}$. Note that some of the edges of $P_{i}$ may not 


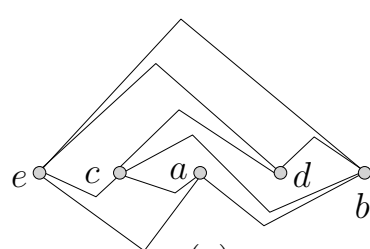

(a)

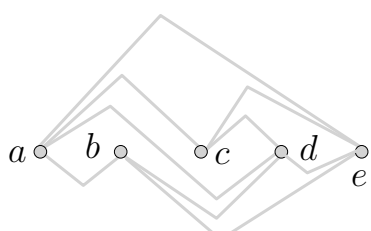

(b)

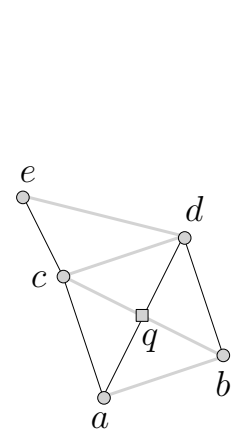

(c)

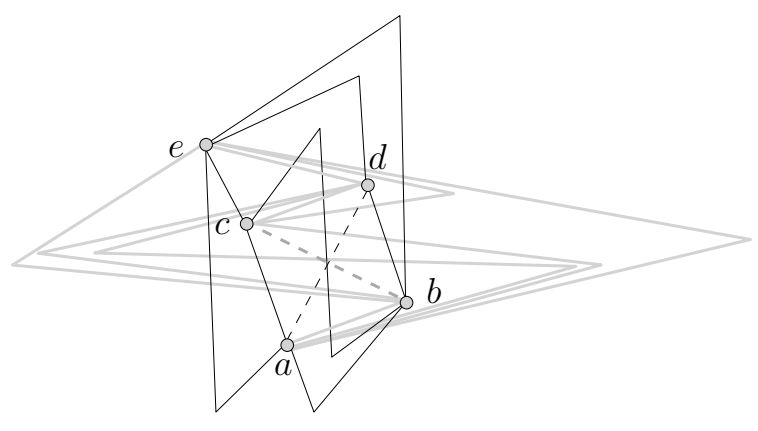

(d)

Figure 2 (a)-(b) Monotone topological book embeddings of $G_{1}$ and $G_{2}$. (c)-(d) Simultaneous embedding of $G_{1}$ and $G_{2}$, where the deleted edges are shown in dashed lines.

exist in $G_{i}$, e.g., edges $(a, d)$ and $(b, c)$ in Figures $2(\mathrm{a})$ and (b), respectively, and these edges of $P_{i}$ create edge crossings in $G_{i}$. Add a dummy vertex at each such edge crossing. Let $\delta_{i}(v)$ be the position of vertex $v$ in $\pi_{i}$. Then $P_{1}$ and $P_{2}$ can be drawn simultaneously on an $O(n) \times O(n)$ grid [5] by placing each vertex at the grid point $\left(\delta_{1}(v), \delta_{2}(v)\right)$; see Figure $2(\mathrm{c})$. The mapping between the dummy vertices of $P_{1}$ and $P_{2}$ can be arbitrary, here we map the dummy vertex on $(a, d)$ to the dummy vertex on $(b, c)$. Finally, the edges of $G_{i}$ that do not belong to $P_{i}$ are drawn. Let $e$ be such an edge in $G_{i}$. If $e$ does not cross the spine, then it is drawn using one bend on one side of $P_{i}$ according to the book embedding of $G_{i}$. Otherwise, let $q$ be a dummy vertex on the edge $e=(u, v)$, which corresponds to the intersection point of $e$ and the spine. The edges $(u, q)$ and $(v, q)$ are drawn on opposite sides of $P_{i}$ such that the polyline from $u$ to $v$ do not create any bend at $q$. Since each of $(u, q)$ and $(v, q)$ contains only one bend, $e$ contains only two bends. Finally, the edges of $P_{i}$ that do not belong to $G_{i}$ are removed from the drawing; see Figure 2(d).

Let $\Gamma$ be a planar polyline drawing of a path $P=\left\{v_{1}, v_{2}, \ldots, v_{n}\right\}$. We call $\Gamma$ an uphill drawing if for any point $q$ on $\Gamma$, the upward ray from $q$ does not intersect the path $v_{1}, \ldots, q$. Note that $q$ may be a vertex location or an interior point of some edge in $\Gamma$. Let $a$ and $b$ be two points in $\mathbb{R}^{2}$. Then $a$ and $b$ are $r$-visible to each other if and only if their exists a polygonal chain of length $r$ with end points $a, b$ that does not intersect $\Gamma$ at any point except possibly at $a, b$. A point $p$ lies between two other points $v, w$, if either the inequality $x(v)<x(p)<x(w)$ or $x(w)<x(p)<x(v)$ holds.

A set of points is monotone if the polyline connecting them from left to right is monotone with respect to $y$-axis. Let $S$ be a set of $n$ points in general position. By the Erdös-Szekeres theorem [14], $S$ can be partitioned into $O(\sqrt{n})$ disjoint monotone subsets, and such a partition can be computed in $O\left(n^{1.5}\right)$ time [2].

\section{Drawing Thickness- $t$ Graphs on $t$ Layers}

In this section we give two separate construction techniques to draw thickness- $t$ graphs on $t$ layers. We first present a construction achieving 2.25n+O(1) upper bound (Section 3.1), which is simple and intuitive. Although the technique is simple, the idea of the construction will be used frequently in the rest of the paper. Therefore, we explained the construction in reasonable details. 
Later, we present a second construction (Section 3.2), which is more involved, and relies on a deep understanding of the geometry of point sets. In this case, the upper bound on the bend complexity will depend on some generalization of Erdös-Szekeres theorem [14], e.g., partitioning a point set into monotone subsequences in higher dimensions (Section 3.2.3).

\subsection{A Simple Construction with Bend Complexity 2.25n+O(1)}

Let $G_{1}, \ldots, G_{t}$ be the planar subgraphs of the input graph $G$, and let $S$ be an ordered set of $n$ points on a semicircular arc. Let $V=\left\{v_{1}, v_{2}, \ldots, v_{n}\right\}$ be the set of vertices of $G$. We show that each $G_{i}$, where $1 \leq i \leq t$, admits a polyline drawing with bend complexity $2.25 n+O(1)$ such that vertex $v_{j}$ is mapped to the $j$ th point of $S$. To draw $G_{i}$, we will use the vertex ordering of its monotone topological book embedding. The following lemma will be useful to draw the spinal path $P_{i}$ of $G_{i}$.

- Lemma 1. Let $S=\left\{p_{0}, p_{1}, \ldots, p_{n+1}\right\}$ be a set of points lying on an $x$-monotone semicircular arc (e.g., see Figure $3(a))$, and let $P=\left\{v_{1}, v_{2}, \ldots, v_{n}\right\}$ be a path of $n$ vertices. Assume that $p_{0}$ and $p_{n+1}$ are the leftmost and rightmost points of $S$, respectively, and the points $p_{1}, \ldots, p_{n}$ are equally spaced between them in some arbitrary order. Then $P$ admits an uphill drawing $\Gamma$ with the vertex $v_{i}$ assigned to $p_{i}$, where $1 \leq i \leq n$, and every point $p_{i}$ satisfies the following properties:

(A) Both the points $p_{0}$ and $p_{n+1}$ are $(3 n / 4)$-visible to $p_{i}$.

(B) One can draw an $x$-monotone polygonal chain from $p_{0}$ to $p_{n+1}$ with $3 n / 4$ bends that intersects $\Gamma$ only at $p_{i}$.

Proof. We prove the lemma by constructing such a drawing $\Gamma$ for $P$. The construction assigns a polyline for each edge of $P$. The resulting drawing may contain edge overlaps, and the bend complexity could be as large as $n-2$. Later we remove these degeneracies and reduce the bend complexity to obtain $\Gamma$.

Drawings of Edges: For each point $p_{i} \in S$, where $1 \leq i \leq n$, we create an anchor point $p_{i}^{\prime}$ at $\left(x\left(p_{i}\right), y\left(p_{i}\right)+\epsilon\right)$, where $\epsilon>0$. We choose $\epsilon$ small enough such that for any $j$, where $1 \leq i \neq j \leq n$, all the points of $S$ between $p_{i}$ and $p_{j}$ lie above $\left(p_{i}^{\prime}, p_{j}^{\prime}\right)$. Figure 3(a) illustrates this property for the anchor point $p_{1}^{\prime}$.

We first draw the edge $\left(v_{1}, v_{2}\right)$ using a straight line segment. For each $j$ from 2 to $n-1$, we now draw the edges $\left(v_{j}, v_{j+1}\right)$ one after another. Assume without loss of generality that $x\left(p_{j}\right)<x\left(p_{j+1}\right)$. We call a point $p \in S$ between $p_{j}$ and $p_{j+1}$ a visited point if the corresponding vertex $v$ appears in $v_{1}, \ldots, v_{j}$, i.e., $v$ has already been placed at $p$. We draw an $x$-monotone polygonal chain $L$ that starts at $v_{j}$, connects the anchors of the intermediate visited points from left to right, and ends at $v_{j+1}$. Figure $3(\mathrm{~b})$ illustrates such a construction.

Since the number of bends on $L$ is equal to the number of visited points of $S$ between $p_{j}$ and $p_{j+1}$, each edge contains at most $\alpha$ bends, where $\alpha$ is the number of points of $S$ between $p_{j}$ and $p_{j+1}$.

Removing Degeneracies: The drawing $D_{n}$ of the path $P$ constructed above contains edge overlaps, e.g., see the edges $\left(v_{3}, v_{4}\right)$ and $\left(v_{4}, v_{5}\right)$ in Figure 3(c). To remove the degeneracies, for each $i$, we spread the corresponding bend points between $p_{i}$ and $p_{i}^{\prime}$, in the order they appear on the path, see Figure 3(d). Consequently, we obtain a planar drawing of $P$. Let the resulting drawing be $D_{n}^{\prime}$. Since each edge $\left(p_{j}, p_{j+1}\right)$ is drawn as an $x$-monotone polyline above the path $p_{1}, \ldots, p_{j}, D_{n}^{\prime}$ satisfies the uphill property. Note that $D_{n}^{\prime}$ may have bend 


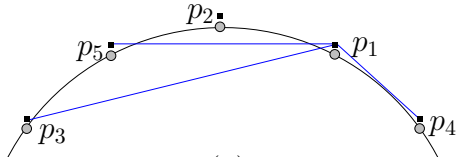

(a)

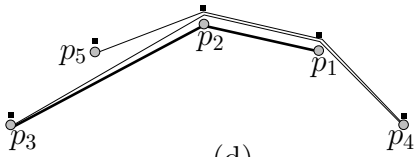

(d)

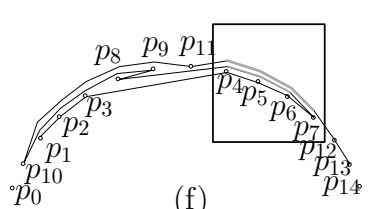

(f)

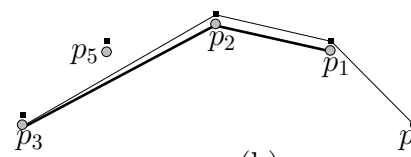

(b)

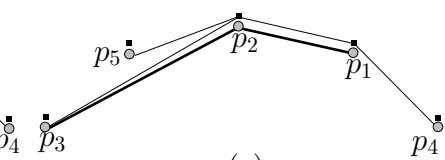

(c)

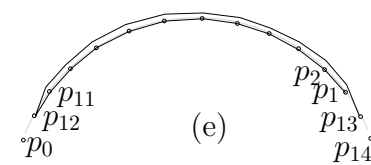

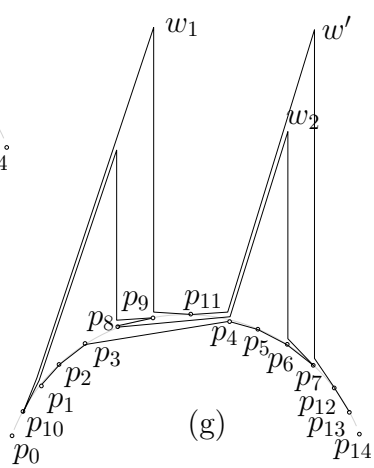

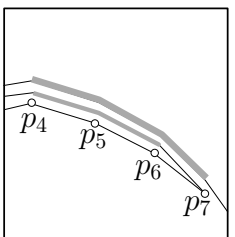

Figure 3 Illustration for the proof of Lemma 1. Anchor points are shown in black squares. For a larger view of this figure, see Appendix A.

complexity $n-2$, e.g., see Figure 3(e). We now show how to reduce the bend complexity and satisfy Properties A-B.

Reducing Bend Complexity: A pair of points in $S$ are consecutive if they do not contain any other point of $S$ in between. Let $e$ be any edge of $P$. Let $C_{e}$ be the corresponding polygonal chain in $D_{n}^{\prime}$. A pair of bend points on $C_{e}$ are called consecutive bends if their corresponding points in $S$ are also consecutive. A bend-interval of $C_{e}$ is a maximal sequence of consecutive bends in $C_{e}$. Note that we can partition the bends on $e$ into disjoint sets of bend-intervals.

For any bend-interval $s$, let $l(s)$ and $r(s)$ be the $x$-coordinates of the left and right endpoints of $s$, respectively. Let $s_{1}$ and $s_{2}$ be two bend-intervals lying on two distinct edges $e_{1}$ and $e_{2}$ in $D_{n}^{\prime}$, respectively, where $e_{2}$ appears after $e_{1}$ in $P$. We claim that the intervals $\left[l\left(s_{1}\right), r\left(s_{1}\right)\right]$ and $\left[l\left(s_{2}\right), r\left(s_{2}\right)\right]$ are either disjoint, or $\left[l\left(s_{1}\right), r\left(s_{1}\right)\right] \subseteq\left[l\left(s_{2}\right), r\left(s_{2}\right)\right]$. We refer to this property as the balanced parenthesis property of the bend-intervals. To verify this property assume that for some $s_{1}, s_{2}$, we have $\left[l\left(s_{1}\right), r\left(s_{1}\right)\right] \cap\left[l\left(s_{2}\right), r\left(s_{2}\right)\right] \neq \phi$. Since $e_{2}$ appears after $e_{1}$, and since $s_{2}$ is a maximal sequence of consecutive bends, the inequalities $l\left(s_{2}\right) \leq l\left(s_{1}\right)$ and $r\left(s_{2}\right) \geq r\left(s_{1}\right)$ hold, i.e., $\left[l\left(s_{1}\right), r\left(s_{1}\right)\right] \subseteq\left[l\left(s_{2}\right), r\left(s_{2}\right)\right]$. We say that $s_{1} i s$ nested by $s_{2}$. Figure $3(\mathrm{f})$ illustrates such a scenario, where $s_{1}, s_{2}$ are shown in thin and thick gray lines, respectively.

We now consider the edges of $P$ in reverse order, i.e., for each $j$ from $n$ to 2 , we modify the drawing of $e=\left(v_{j}, v_{j-1}\right)$. For each bend-interval $s=\left(b_{1}, b_{2} \ldots, b_{r}\right)$ of $C_{e}$, if $s$ has three or more bends, then we delete the bends $b_{2}, \ldots, b_{r-1}$, and join $b_{1}$ and $b_{r}$ using a new bend point $w$. To create $w$, we consider the two cases of the balanced parenthesis property.

If $s$ is not nested by any other bend-interval in $D_{n}^{\prime}$, then we place $w$ high enough above $b_{r}$ such that the chain $b_{1}, w, b_{r}$ does not introduce any edge crossing, e.g., see the point $w_{1}(=w)$ in Figure $3(\mathrm{~g})$. On the other hand, if $s$ is nested by some other bend-interval, then let $s^{\prime}$ be such a bend-interval immediately above $s$. Since $s^{\prime}=\left(b_{1}^{\prime}, b_{2}^{\prime}, \ldots, b_{r}^{\prime}\right)$ is already processed, it must have been replaced by some chain $b_{1}^{\prime}, w^{\prime}, b_{r}^{\prime}$. Therefore, we can find a location for $b$ inside $\angle b_{1}^{\prime} w^{\prime} b_{r}^{\prime}$ such that the chain $b_{1}, w, b_{r}$ does not introduce any edge crossing, e.g., see the points $w^{\prime}$ and $w_{2}(=w)$ in Figure $3(\mathrm{~g})$. Let the resulting drawing of $P$ be $\Gamma$. 
We now show that the above modification reduces the bend complexity to $3 n / 4$. Let $e$ be an edge of $P$ that contains $\alpha$ points from $S$ between its endpoints. Let $C_{e}$ be the corresponding polygonal chain in $D_{n}^{\prime}$. Recall that any bend-interval of length $\ell$ in $C_{e}$ contributes to $\min \{\ell, 3\}$ bends on $e$ in $\Gamma$. Therefore, if there are at most $\alpha / 4$ bend-intervals on $C_{e}$, then $e$ can have at most $3 \alpha / 4$ bends in $\Gamma$. Otherwise, if there are more than $\alpha / 4$ bend-intervals, then there are at least $\alpha / 4$ points $^{1}$ of $S$ that do not contribute to bends on $C_{e}$. Therefore, in both cases, $C_{e}$ can have at most $3 \alpha / 4$ bends in $\Gamma$.

Satisfying Properties A-B: Let $p_{i}$ be any point of $S \backslash\left\{p_{0}, p_{n+1}\right\}$. We first show that $p_{0}$ is $(3 n / 4)$-visible to $p_{i}$. Let $D_{i}$, where $1 \leq i \leq n$, be the drawing of the path $v_{1}, v_{2}, \ldots, v_{i}$. Observe that one can insert an edge $\left(p_{0}, p_{i}\right)$ using an $x$-monotone polyline $L$ such that the bends on $L$ correspond to the intermediate visited points. Now the drawing of the rest of the path $v_{i}, v_{i+1}, \ldots, v_{n}$ can be continued such that it does not cross $L$. Therefore, if the number of points of $S$ between $p_{0}$ and $p_{i}$ is $\alpha$, then $L$ has at most $\alpha$ bends. Finally, the process of reducing bend complexity improves the number of bends on $L$ to $3 \alpha / 4$.

Similarly, we can observe that $p_{n+1}$ is at most $3 \alpha^{\prime} / 4$ visible to $p_{i}$, where $\alpha^{\prime}$ is the number of points of $S$ between $p_{i}$ and $p_{n+1}$. Since the edges $\left(p_{0}, p_{i}\right)$ and $\left(p_{i}, p_{n+1}\right)$ are $x$-monotone, we can draw an $x$-monotone polygonal chain from $p_{0}$ to $p_{n+1}$ with at most $3\left(\alpha+\alpha^{\prime}\right) / 4 \leq(3 n / 4)$ bends that intersects $\Gamma$ only at $p_{i}$.

- Theorem 2. Every n-vertex graph of $t$ admits a drawing on $t$ layers with bend complexity $2.25 n+O(1)$.

Proof. Let $G_{1}, \ldots, G_{t}$ be the planar subgraphs of the input graph $G$, and let $V=\left\{v_{1}, v_{2}\right.$, $\left.\ldots, v_{n}\right\}$ be the set of vertices of $G$. Let $S=\left\{p_{0}, p_{1}, \ldots, p_{n+1}\right\}$ be a set of $n+2$ points lying on a semicircular arc as defined in Lemma 1. Let $P_{i}$ be spinal path of the monotone topological book embedding of $G_{i}$, where $1 \leq i \leq t$. We first compute an uphill drawing $\Gamma_{i}$ of the path $P_{i}$. We then draw the edges of $G_{i}$ that do not belong to $P_{i}$. Let $e=(u, v)$ be such an edge, and without loss of generality assume that $u$ appears to the left of $v$ on the spine.

If $e$ lies above (resp., below) the spine, then we draw two $x$-monotone polygonal chains; one from $u$ to $p_{0}$ (resp., $p_{n+1}$ ), and the other from $v$ to $p_{0}$ (resp., $p_{n+1}$ ). By Lemma 1 , these polygonal chains do not intersect $\Gamma_{i}$ except at $u$ and $v$, and each contains at most $3 n / 4$ bends. Hence $e$ contains at most $1.5 n$ bends in total.

If $e$ crosses the spine, then it crosses some edge $\left(w, w^{\prime}\right)$ of $P_{i}$. Draw the edges $(u, w)$ and $(w, v)$ using the polylines $u, \ldots, p_{0}, \ldots, w$ and $w, \ldots, p_{n+1}, \ldots, v$, respectively. The polylines $u, \ldots, p_{0}$ and $p_{n+1}, \ldots, v$ are $x$-monotone, and have at most $3 n / 4$ bends each. The polyline $C=\left(p_{0}, \ldots, w \ldots, p_{n+1}\right)$ is also $x$-monotone and has at most $3 n / 4$ bends. Hence the number of bends is $2.25 n$ in total. It is straightforward to avoid the degeneracy at $w$, by adding a constant number of bends on $C$.

Note that we still have some edge overlaps at $p_{0}$ and $p_{n+1}$. It is straightforward to remove these degeneracies by adding only a constant number of more bends per edge.

\subsection{A Construction for Small Values of $t$}

In this section we give another construction to draw thickness- $t$ graphs on $t$ layers. We first show that every thickness- $t$ graph, where $t \in\{3,4\}$, can be drawn on $t$ layers with bend complexity $O(\sqrt{n})$, and then show how to extend the technique for larger values of $t$.

1 Every pair of consecutive bend-intervals contain such a point in between. 


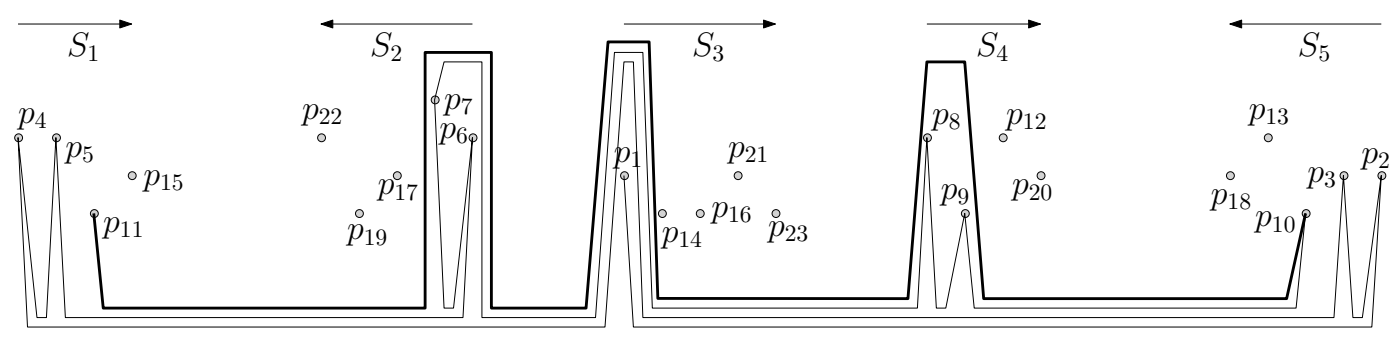

Figure 4 Illustration for the proof of Lemma 3 . The edge $\left(p_{10}, p_{11}\right)$ is shown in bold. Passing through each intermediate set requires at most 4 bends.

\subsubsection{Construction when $t=3$}

Let $S$ be an ordered set of $n$ points, where the ordering is by increasing $x$-coordinate. A $(k, n)$ group $S_{k, n}$ is a partition of $S$ into $k$ disjoint ordered subsets $\left\{S_{1}, \ldots, S_{k}\right\}$, each containing contiguous points from $S$. Label the points of $S$ using a permutation of $p_{1}, p_{2}, \ldots, p_{n}$ such that for each set $S^{\prime} \in S_{k, n}$, the indices of the points in $S^{\prime}$ are either increasing or decreasing. If the indices are increasing (resp., decreasing), then we refer $S^{\prime}$ as a rightward (resp., leftward) set. We will refer to such a labelling as a smart labelling of $S_{k, n}$. Figure 4 illustrates a $(5,23)$-group and a smart labelling of the underlying point set $S_{5,23}$.

Note that for any $i$, where $1 \leq i \leq n$, deletion of the points $p_{1}, \ldots, p_{i}$ removes the points of the rightward (resp., leftward) sets from their left (resp., right). The necklace of $S_{k, n}$ is a path obtained from a smart labelling of $S_{k, n}$ by connecting the points $p_{i}, p_{i+1}$, where $1 \leq i \leq n-1$. The following lemma constructs an uphill drawing of the necklace using $O(k)$ bends per edge.

- Lemma 3. Let $S$ be a set of $n$ points ordered by increasing $x$-coordinate, and let $S_{k, n}=$ $\left\{S_{1}, \ldots, S_{k}\right\}$ be a $(k, n)$-group of $S$. Label $S_{k, n}$ with a smart labelling. Then the necklace of $S_{k, n}$ admits an uphill drawing with $O(k)$ bends per edge.

Proof. We construct this uphill drawing incrementally in a similar way as in the proof of Lemma 1. Let $D_{j}$, where $1 \leq j \leq n$, be the drawing of the path $p_{1}, \ldots, p_{j}$. At each step of the construction, we maintain the invariant that $D_{j}$ is an uphill drawing.

We first assign $v_{1}$ to $p_{1}$. Then for each $i$ from 1 to $n-1$, we draw the edge $\left(p_{i}, p_{i+1}\right)$ using an $x$-monotone polyline $L$ that lies above $D_{i}$ and below the points $p_{j^{\prime}}$, where $j^{\prime}>i+1$. Figure 4 illustrates such a drawing of $\left(p_{i}, p_{i+1}\right)$.

The crux of the construction is that one can draw such a polyline $L$ using at most $O(k)$ bends. Assume that $p_{i}$ and $p_{i+1}$ belong to the sets $S_{l} \in S_{k, n}$ and $S_{r} \in S_{k, n}$, respectively. If $S_{l}$ and $S_{r}$ are identical, then $p_{i}$ and $p_{i+1}$ are consecutive, and hence it suffices to use at most $O(1)$ bends to draw $L$. On the other hand, if $S_{l}$ and $S_{r}$ are distinct, then there can be at most $k-2$ sets of $S_{k, n}$ between them. Let $S_{m}$ be such a set. While passing through $S_{m}$, we need to keep the points that already belong to the path, below $L$, and the rest of the points above $L$. By the property of smart labelling, the points that belong to $D_{i}$ are consecutive in $S_{m}$, and lie to the left or right side of $S_{m}$ depending on whether $S_{m}$ is rightward or leftward. Therefore, we need only $O(1)$ bends to pass through $S_{m}$. Since there are at most $k-2$ sets between $S_{l}$ and $S_{r}, O(k)$ bends suffice to construct $L$.

We are now ready to describe the main construction. Let $G$ be an $n$-vertex thickness-3 graph, and let $G_{1}, G_{2}, G_{3}$ be the planar subgraphs of $G$. Let $P_{i}$ be the spinal path of the monotone topological book embedding of $G_{i}$, where $1 \leq i \leq 3$. We first create a set of $n$ points and assign them to the vertices of $G$. Later we route the edges of $G$. 


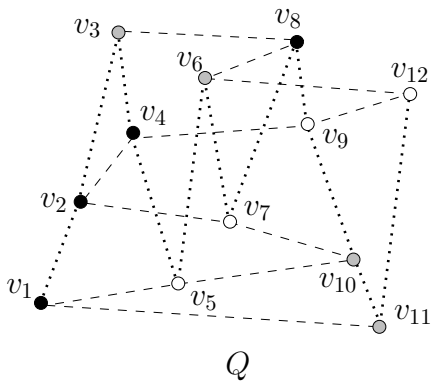

(a)

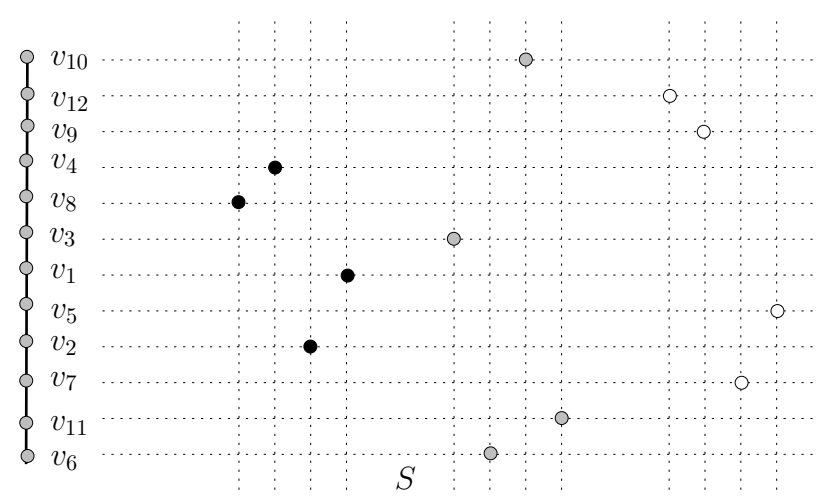

(b)

Figure 5 Creating vertex locations for drawing thickness-3 graphs, where $P_{1}, P_{2}$ and $P_{3}$ are shown in dotted, dashed and thick solid lines, respectively.

Creating Vertex Locations: Assume without loss of generality that $P_{1}=\left(v_{1}, \ldots, v_{n}\right)$. For each $i$ from 1 to $n$, we place a point at $(i, j)$ in the plane, where $j$ is the position of $v_{j}$ in $P_{2}$. Let the resulting point set be $Q$. Recall that $Q$ can be partitioned into disjoint monotone subsets $Q_{1}, \ldots, Q_{k}$, where $k \in O(\sqrt{n})$ [2]. Figure $5($ a) illustrates such a partition in black, gray and white.

The sets $Q_{1}, \ldots, Q_{k}$ are ordered by the $x$-coordinate, and the indices of the labels of the points at each set is in increasing order. Therefore, if we place the points of the $i$ th set between the lines $x=2(i-1) n$ and $x=(2 i-1) n$, then the resulting point set $Q^{\prime}$ would be a $(k, n)$-group, labelled by a smart labelling. Finally, we adjust the $y$-coordinates of the points according to the position of the corresponding vertices in $P_{3}$. Let the resulting point set be $S$. Figure 5(b) illustrates the vertex locations, where $P_{1}=\left(v_{1}, v_{2}, \ldots, v_{n}\right), P_{2}=\left(v_{11}, v_{1}, \ldots, v_{3}\right)$, and $P_{3}=\left(v_{6}, v_{11}, \ldots, v_{10}\right)$.

Edge Routing: It is straightforward to observe that the path $P_{1}$ is a necklace for the current labelling of the points of $S_{k, n}$. Therefore, by Lemma 3, we can construct an uphill drawing of $P_{1}$ on $S$. Observe that for every set $S^{\prime} \in S_{k, n}$, the corresponding points are monotone in $Q$, i.e., the points of $S^{\prime}$ are ordered along the $x$-axis either in increasing or decreasing order of their $y$-coordinates in $Q$. Therefore, relabelling the points according to the increasing order of their $y$-coordinates in $Q$ will produce another smart labelling of $S$, and the corresponding necklace would be the path $P_{2}$. Therefore, we can use Lemma 3 to construct an uphill drawing of $P_{2}$ on $S$. Since the height of the points of $S$ are adjusted according to the vertex ordering on $P_{3}$, connecting the points of $S$ from top to bottom with straight line segments yields a $y$-monotone drawing of $P_{3}$.

We now route the edges of $G_{i}$ that do not belong to $P_{i}$, where $1 \leq i \leq 3$. Since $P_{3}$ is drawn as a $y$-monotone polygonal path, we can use the technique of Erten and Kobourov [15] to draw the remaining edges of $G_{3}$. To draw the edges of $G_{2}$, we insert two points $p_{0}$ and $p_{n+1}$ to the left and right of all the points of $S$, respectively. Then the drawing of the remaining edges of $G_{1}$ and $G_{2}$ is similar to the edge routing described in the proof of Theorem 2 . That is, if the edge $e=(u, v)$ lies above (resp., below) the spine, then we draw it using two $x$-monotone polygonal chains from $p_{0}$ (resp., $p_{n+1}$ ). Otherwise, if $e$ crosses the spine, then we draw three $x$-monotone polygonal chains, one from $u$ to $p_{0}$, another from $p_{0}$ to $p_{n+1}$, and the third one from $v$ to $p_{n+1}$. Since $k \in O(\sqrt{n})$, the number of bends on $e$ is $O(\sqrt{n})$. Finally, we remove the degeneracies, which increases the bends per edge by a small constant. 


\subsubsection{Construction when $t=4$}

We now show that the technique for drawing thickness-3 graphs can be generalized to draw thickness-4 graphs with the same bend complexity.

Let $G_{1}, \ldots, G_{4}$ be the planar subgraphs of $G$, and let $P_{1}, \ldots, P_{4}$ be the corresponding spinal paths. While constructing the vertex locations, we use a new $y$-coordinate assignment for the points of $S$. Instead of placing the points according to the vertex ordering on the path $P_{3}$, we create a particular order, by transposing the $x$ - and $y$-axis, that would help to construct uphill drawings of $P_{3}$ and $P_{4}$ with bend complexity $O(\sqrt{n})$. That is, we first create a $\left(k^{\prime}, n\right)$-group $S_{k^{\prime}, n}^{\prime}$ using $P_{3}$ and $P_{4}$, where $k^{\prime} \in O(\sqrt{n})$, in a similar way that we created $S_{k, n}$ using $P_{1}$ and $P_{2}$. We then adjust the $y$-coordinates of the points of $S$ according to the order these points appear in $S_{k^{\prime}, n}^{\prime}$. Appendix B includes an example of such a construction.

The construction of $G_{1}$ and $G_{2}$ remains the same as described in the previous section. However, since $P_{3}$ and $P_{4}$ now admit uphill drawings on $S$ with respect to $y$-axis, the drawings of $G_{3}$ and $G_{4}$ are now analogous to the construction of $G_{1}$ and $G_{2}$.

\subsubsection{Construction when $t>4$}

De Bruijn [19] observed that the result of Erdös-Szekeres [14] can be generalized to higher dimensions. Given a sequence $\rho$ of $n$ tuples, each of size $\kappa$, one can find a subsequence of at least $n^{1 / \lambda}$ tuples, where $\lambda=2^{\kappa}$, such that they are monotone (i.e., increasing or decreasing) in every dimension. If we repeatedly extract such monotone sequences, then we obtain a partition of $\rho$ into a set of monotone subsequences. We use this idea to extend our drawing algorithm to higher thickness.

Let $G_{1}, \ldots, G_{t}$ be the planar subgraphs of $G$, and let $P_{1}, \ldots, P_{t}$ be the corresponding spinal paths. Let $v_{1}, v_{2}, \ldots, v_{n}$ be the vertices of $G$. Construct a corresponding sequence $\rho=\left(\tau_{1}, \tau_{2}, \ldots, \tau_{n}\right)$ of $n$ tuples, where each tuple is of size $t$, and the $i$ th element of a tuple $\tau_{j}$ corresponds to the position of the corresponding vertex $v_{j}$ in $P_{i}$, where $1 \leq i \leq t$ and $1 \leq j \leq n$. We now partition $\rho$ into a set of monotone subsequences. Let $f(n, t)$ be the number of monotone subsequences in this partition.

For each of these monotone sequences, we create an ordered set of consecutive points along the $x$-axis, where the vertex $v_{j}$ corresponds to the point $p_{j}$. It is now straightforward to observe that these sets correspond to a $(k, n)$-group $S_{k, n}$, where $k \leq f(n, t)$. Furthermore, since each group corresponds to a monotone sequence of tuples, for each $P_{i}$, the positions of the corresponding vertices are either increasing or decreasing. Hence, every path $P_{i}$ corresponds to a necklace for some smart labelling of $S_{k, n}$. Therefore, by Lemma 3, we can construct an uphill drawing of $P_{i}$ on $S$. We now add the remaining edges of $G_{i}$ following the construction described in Section 3.2.1. Since $k \leq f(n, t)$, the number of bends is bounded by $O(f(n, t))$.

Observe that all the points in the above construction have the same $y$-coordinate. Therefore, we can improve the construction by distributing the load equally among the $x$-axis and $y$-axis as we did in Section 3.2.2. Specifically, we draw the graphs $G_{1}, \ldots, G_{\lceil t / 2\rceil}$ using the uphill drawings of their spinal paths with respect to the $x$-axis, and the remaining graphs using the uphill drawings of their spinal paths with respect to the $y$-axis. Consequently, the bend complexity decreases to $O(f(n,\lceil t / 2\rceil))$. We can improve this bound further by observing that we are free to choose any arbitrary vertex labelling for $G$ while creating the initial sequence of tuples. Instead of using an arbitrary labelling, we could label the vertices according to their ordering on some spinal path, which would reduce the bend complexity to $O(f(n,\lceil(t-2) / 2\rceil))$. As shown in Sections 3.2.1 and 3.2.2, if $t \in\{3,4\}$, then $f(n,\lceil(t-2) / 2\rceil) \in O(\sqrt{n})$. 
- Theorem 4. Every n-vertex graph $G$ of thickness $t \geq 3$ admits a drawing on $t$ layers with bend complexity $O(\beta)$, where $\beta$ is the minimum integer such that the sequence of $n$ tuples obtained from the spinal paths of $G$ can be partitioned into $\beta$ monotone subsequences. Furthermore, if $t \in\{3,4\}$, then $\beta \in O(\sqrt{n})$.

A careful analysis of the generalization of Erdös-Szekeres [14] theorem gives an $O\left(\sqrt{2}^{t}\right.$. $\left.n^{1-(1 / \gamma)}\right)$ upper bound on the bend complexity, where $\gamma=2^{\lceil(t-2) / 2\rceil}$. For the details, we refer to the full version of the paper [11].

\section{Drawing Graphs of Linear Arboricity $k$}

In this section we construct polyline drawings, where the layer number and bend complexities are functions of the linear arboricity of the input graphs. We show that the bandwidth of a graph can be bounded in terms of its linear arboricity and the number of vertices, and then the result follows from an application of Lemma 1.

The bandwidth of an $n$-vertex graph $G=(V, E)$ is the minimum integer $b$ such that the vertices can be labelled using distinct integers from 1 to $n$ satisfying the condition that for any edge $(u, v) \in E$, the absolute difference between the labels of $u$ and $v$ is at most $b$. The following lemma proves an upper bound on the bandwidth of graphs.

- Lemma 5. Given an n-vertex graph $G=(V, E)$ with linear arboricity $k$, the bandwidth of $G$ is at most $\frac{3(k-1) n}{(4 k-2)}$.

Proof. Without loss of generality assume that $G$ is a union of $k$ spanning paths $P_{1}, \ldots, P_{k}$. For any ordered sequence $\sigma$, let $\sigma(i)$ be the element at the $i$ th position, and let $|\sigma|$ be the number of elements in $\sigma$. We now construct an ordered sequence $\sigma=\sigma_{1} \circ \sigma_{2} \circ \ldots \circ \sigma_{k} \circ \sigma_{k+1}$ of the vertices in $V$, as follows.

$\sigma_{1}$ : We initially place the first $x$ vertices of $P_{1}$ in the sequence, where the exact value of $x$ is to be determined later.

$\sigma_{2}$ : We then place the vertices that are neighbors of $\sigma_{1}$ in $P_{2}$, in order, i.e., we first place the neighbors of $\sigma_{1}(1)$, then the neighbors of $\sigma_{1}(2)$ that have not been placed yet, and so on. $\sigma_{i}$ : For each $i=3, \ldots, k$, we place the vertices that are neighbors of $\sigma_{1}$ in $P_{i}$ in order.

$\sigma_{k+1}$ : We next place the remaining vertices of $P_{1}$ in order.

Figure 6(a) illustrates an example for three paths with $x=2$. Observe that $\left|\sigma_{1}\right| \leq x$, and $\left|\sigma_{t}\right| \leq 2 x$, where $1<t \leq k$. We now compute an upper bound on the bandwidth of $G$ using the vertex ordering of $\sigma$.

For any $i, j$, where $1 \leq i<j \leq k+1$, let $\sigma_{i, j}$ be the sequence $\sigma_{i} \circ \ldots \circ \sigma_{j}$. The edges of $P_{1}$ that are in $\sigma_{1}$ have bandwidth 1 , and those that are in $\sigma_{1}(x) \circ \sigma_{2, k+1}$ have bandwidth at most $(n-x)$, e.g., see Figure $6(\mathrm{~b})$. Now let $(v, w)$ be an edge of $G$ that does not belong to $P_{1}$. We compute the bandwidth of $(v, w)$ considering the following cases.

Case 1. If none of $v$ and $w$ belongs to $\sigma_{1}$, then the bandwidth of $(v, w)$ is at most $(n-x)$. Case 2. If both $v$ and $w$ belong to $\sigma_{1}$, then the bandwidth of $(v, w)$ is at most $x$.

Case 3. If at most one of $v$ and $w$ belongs to $\sigma_{1}$, then without loss of generality assume that $v$ belongs to $\sigma_{1}$. Since $(v, w)$ does not belong to $P_{1}$, we may assume that $w$ belongs to the path $P_{t}$, where $1<t \leq k$. By the construction of $\sigma, w$ belongs to $\sigma_{1, t}$, e.g., see Figure $6(\mathrm{~b})$. Without loss of generality assume that $w$ belongs to $\sigma_{r}$, where $1<r \leq t$. Let $v$ be the $q$ th vertex in the sequence $\sigma$. Then the position of $w$ cannot be more than $q+2 x \cdot(r-2)+2 q$, where the term $2 x \cdot(r-2)$ corresponds to the length of $\sigma_{2} \circ \ldots \circ \sigma_{r-1}$. Therefore, the bandwidth of the edge $(v, w)$ is at most $2 x \cdot(r-2)+2 q \leq 2 x(r-1) \leq 2 x(t-1)$. 


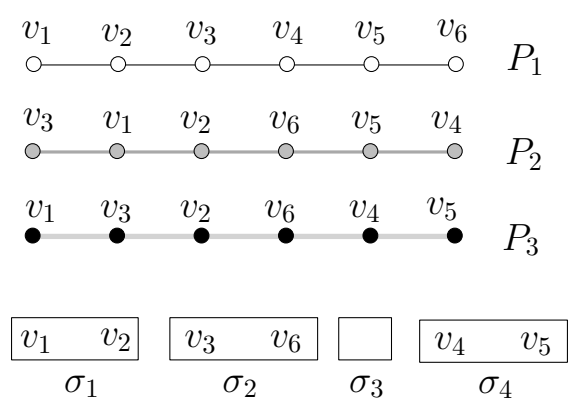

(a)

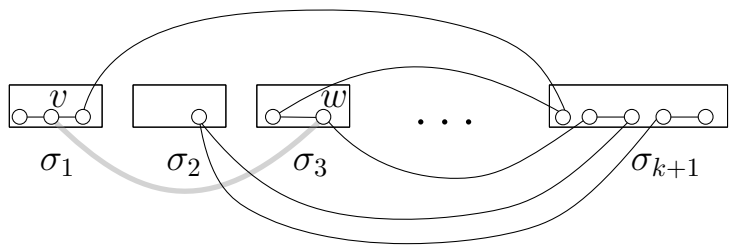

(b)

Figure 6 (a) Construction of $\sigma$. (b) A schematic representation of $P_{1}$ and $(v, w)$, where $(v, w)$ belongs to $P_{3}$.

Observe that the bandwidth of the edges of $P_{1}$ is upper bounded by $(n-x)$. The bandwidth of any edge that belongs to $P_{t}$, where $1<t \leq k$ is at most $\max \{n-x, 2 x(k-1)\}$. Consequently, the bandwidth of $G$ is at $\operatorname{most} \max \{n-x, 2 x(k-1)\} \leq \frac{(2 k-2) n}{(2 k-1)}$, where $x=\frac{n}{(2 k-1)}$.

The following theorem is immediate from the proof of Lemmas 1 and 5 .

- Theorem 6. Every $n$-vertex graph with linear arboricity $k$ can be drawn on $k$ layers with at most $\frac{3(k-1) n}{(4 k-2)}<0.75 n$ bends per edge.

\section{Conclusions}

In this paper we have developed algorithms to draw graphs on few planar layers and with low bend complexity. Although our algorithms do not construct drawings with integral coordinates, it is straightforward to see that these drawings can also be constructed on polynomial-size integer grids, where all vertices and bends have integral coordinates. We leave the task of finding compact grid drawings achieving the same upper bounds as a direction for future research.

We believe our upper bounds on bend complexity to be nearly tight, but we require more evidence to support this intuition. The only related lower bound is that of Pach and Wenger [20], who showed that given a planar graph $G$ and a unique location to place each vertex of $G, \Omega(n)$ bends are sometimes necessary to construct a planar polyline drawing of $G$ with the given vertex locations. Therefore, a challenging research direction would be to prove tight lower bounds on the bend complexity while drawing thickness- $t$ graphs on $t$ layers.

Acknowledgement. We thank anonymous reviewers for many constructive comments and suggestions.

\section{References}

1 Melanie Badent, Emilio Di Giacomo, and Giuseppe Liotta. Drawing colored graphs on colored points. Theoretical Computer Science, 408(2-3):129-142, 2008.

2 Reuven Bar-Yehuda and Sergio Fogel. Partitioning a sequence into few monotone subsequences. Acta Informatica, 35(5):421-440, 1998. 
3 János Barát, Jiří Matoušek, and David R. Wood. Bounded-degree graphs have arbitrarily large geometric thickness. Electronic Journal of Combinatorics, 13(R3), 2006.

4 Thomas Bläsius, Stephen G. Kobourov, and Ignaz Rutter. Simultaneous embedding of planar graphs. In Roberto Tamassia, editor, Handbook of Graph Drawing and Visualization, chapter 11, pages 349-380. CRC Press, August 2013.

5 Peter Braß, Eowyn Cenek, Christian A. Duncan, Alon Efrat, Cesim Erten, Dan Ismailescu, Stephen G. Kobourov, Anna Lubiw, and Joseph S. B. Mitchell. On simultaneous planar graph embeddings. Computational Geometry, 36(2):117-130, 2007.

6 Michael B. Dillencourt, David Eppstein, and Daniel S. Hirschberg. Geometric thickness of complete graphs. Journal of Graph Algorithms and Applications, 4(3):5-17, 2000.

7 Vida Dujmović and David R. Wood. Graph treewidth and geometric thickness parameters. Discrete E Computational Geometry, 37(4):641-670, 2007.

8 Christian A. Duncan. On graph thickness, geometric thickness, and separator theorems. Computational Geometry, 44(2):95-99, 2011.

9 Christian A. Duncan, David Eppstein, and Stephen G. Kobourov. The geometric thickness of low degree graphs. In Proceedings of the 20th ACM Symposium on Computational Geometry (SoCG), pages 340-346. ACM, 2004.

10 Stephane Durocher, Ellen Gethner, and Debajyoti Mondal. Thickness and colorability of geometric graphs. Computational Geometry: Theory and Applications, 56:1-18, 2016.

11 Stephane Durocher and Debajyoti Mondal. Relating graph thickness to planar layers and bend complexity, 2016. URL: http://arxiv.org/abs/1602.07816.

12 Hikoe Enomoto and Miki Shimabara Miyauchi. Embedding graphs into a three page book with $O(m \log n)$ crossings of edges over the spine. SIAM Journal on Discrete Mathematics, 12(3):337-341, 1999.

13 David Eppstein. Separating thickness from geometric thickness. In János Pach, editor, Towards a Theory of Geometric Graphs. American Mathematical Society, 2004.

14 Paul Erdös and George Szekeres. A combinatorial theorem in geometry. Compositio Math., 2:463-470, 1935.

15 Cesim Erten and Stephen G. Kobourov. Simultaneous embedding of planar graphs with few bends. Journal of Graph Algorithms and Applications, 9(3):347-364, 2005.

16 István Fáry. On straight-line representation of planar graphs. Acta Sci. Math. (Szeged), 11:229-233, 1948.

17 Emilio Di Giacomo and Giuseppe Liotta. Simultaneous embedding of outerplanar graphs, paths, and cycles. International Journal of Computational Geometry $\&$ Applications, 17(2):139-160, 2007.

18 Taylor Gordon. Simultaneous embeddings with vertices mapping to pre-specified points. In Proceedings of the 18th Annual International Conference on Computing and Combinatorics (COCOON), volume 7434 of LNCS, pages 299-310. Springer, 2012.

19 Joseph B. Kruskal. Monotonic subsequences. Proceedings of the American Mathematical Society, 4:264-274, 1953.

20 János Pach and Rephael Wenger. Embedding planar graphs at fixed vertex locations. Graphs $\mathscr{G}$ Combinatorics, 17(4):717-728, 2001.

21 David R. Wood. Geometric thickness in a grid. Discrete Mathematics, 273(1-3):221-234, 2003. 